\title{
Two putative MAP kinase genes, ZrHOG1 and ZrHOG2, cloned from the salt-tolerant yeast Zygosaccharomyces rouxii are functionally homologous to the Saccharomyces cerevisiae HOG1 gene
}

\author{
Tomoko Iwaki, Youichi Tamai and Yasuo Watanabe
}

Author for correspondence: Yasuo Watanabe. Tel: +81 89946 9849. Fax: +81899774364.
e-mail: watanabe@agr.ehime-u.ac.jp

Laboratory of Biochemistry, Faculty of Agriculture, Ehime University, 3-5-7 Tarumi, Matsuyama, Ehime 790-8566, Japan

\begin{abstract}
The salt-tolerant yeast Zygosaccharomyces rouxii can adjust its osmotic balance when responding to osmotic shock by accumulating glycerol as the compatible osmolyte. However, the mechanism of glycerol production in Z. rouxii cells and its genetic regulation remain to be elucidated. Two putative mitogen-activated protein (MAP) kinase genes, ZrHOG1 and ZrHOG2, were cloned from Z. rouxii by their homology with HOG1 from Saccharomyces cerevisiae. The deduced amino acid sequences of ZrHog1p and ZrHog2p indicated close homology to that of Hog1p and contained a TGY motif for phosphorylation by MAP kinase kinase. When ZrHOG1 or ZrHOG2 was expressed in an S. cerevisiae hog $1 \Delta$ null mutant, the salt tolerance and osmotic tolerance characteristics of wild-type S. cerevisiae were restored. In addition, the aberrant cell morphology and low glycerol content of the hog1 $1 \Delta$ null mutant were corrected, indicating that ZrHog1p and ZrHog2p have functions similar to Hog1p. While the transcription of the glycerol-3-phosphate dehydrogenase gene (GPD1) of the ZrHOG1-harbouring S. cerevisiae mutant was similar to that of wild-type S. cerevisiae, the ZrHOG2-harbouring strain showed prolonged GPD1 transcription. Both Zrhog1 1 and Zrhog2 Z . rouxii null mutants showed a decrease in salt tolerance compared to the wild-type strain. The present study suggested the presence of a high-osmolarity glycerol response (HOG) pathway in Z. rouxii similar to that elucidated in S. cerevisiae. Two putative MAP kinase genes in $Z$. rouxii appeared to be significant in either osmotic regulation or ion homeostasis.
\end{abstract}

Keywords: Zygosaccharomyces rouxii, salt tolerance, MAP kinase gene, glycerol production, glycerol-3-phosphate dehydrogenase gene

\section{INTRODUCTION}

In Saccharomyces cerevisiae, hyperosmotic shock induces the enhanced formation and accumulation of glycerol as a compatible osmolyte (Reed et al., 1987). This protects against osmotic lysis. Recently, the osmotic stress signal transmission pathway of $S$. cerevisiae has been elucidated, and was named the high-osmolarity glycerol response (HOG) pathway (Brewster et al.,

Abbreviations: GPDH, glycerol-3-phosphate dehydrogenase; HOG, highosmolarity glycerol response; MAP, mitogen-activated protein.

The GenBank/EMBLDDBJ accession numbers for the sequences in this paper are AB012146 (ZrHOG1) and AB012088 (ZrHOG2).
1993). It belongs to the mitogen-activated protein (MAP) kinase cascade (Brewster et al., 1993).

In the HOG pathway of $S$. cerevisiae, there are two osmosensors (encoded by SLN1 and SHO1 genes) present in the plasma membrane (Ota \& Varshavsky, 1993; Maeda et al., 1994). The osmotic stress signal sensed by the $S L N 1$ product $(S \ln 1 \mathrm{p})$ is, in turn, transmitted from Ypd1p to Ssk1p to Ssk2/22p (MAP kinase kinase kinase) to Pbs2p (MAP kinase kinase) and then finally to Hog1p (MAP kinase) (Posas et al., 1996). On the other hand, Sho1p (Maeda et al., 1995) regulates the action of Hog1p via Ste11p (MAP kinase kinase kinase) and Pbs $2 p$ (Posas \& Saito, 1997). The activated Hog $1 p$ 
directly or indirectly enhances the activity of a transcriptional regulator which induces transcription of stress protein genes harbouring stress regulatory elements (STRE) in their promoters (Schuller et al., 1994). Since the glycerol-3-phosphate dehydrogenase (GPDH) gene (GPD1) harbours four STREs in the 5'flanking region, the transcription of GPD1 is enhanced and the enzymic activity increases under hyperosmotic conditions (Albertyn et al., 1994; Akhtar et al., 1997; Norbeck \& Blomberg, 1997). As a result, the intracellular concentration of glycerol rises and the osmotic balance is adjusted.

The salt-tolerant yeast Zygosaccharomyces rouxii also accumulates glycerol intracellularly as the main compatible osmolyte in response to hyperosmotic shock (Reed et al., 1987). The participation of GPDH in the production of glycerol in $Z$. rouxii cells is not obvious. Moreover, the transmission pathway of the osmotic stress signal has not yet been elucidated. Thus, in the present study, to clarify whether a similar pathway to that elucidated in $S$. cerevisiae is present in $Z$. rouxii, two $Z$. rouxii genes with homology to HOG1 were cloned and the functions of the cloned genes were analysed in $S$. cerevisiae hog1s null mutants.

\section{METHODS}

Strains and cultures. A salt-tolerant yeast, Z. rouxii strain MA11-3 [from Dr Ushio, Higashimaru Shoyu Co. Ltd, Hyogo, Japan; MAT $\propto$ leu2 (pSR1)], was cultured aerobically at $30^{\circ} \mathrm{C}$ in YPAD medium $(0.5 \%, \mathrm{w} / \mathrm{v}$, yeast extract; $2 \%, \mathrm{w} / \mathrm{v}$, polypeptone; $2 \%, \mathrm{w} / \mathrm{v}$, glucose; $0.5 \%$, w/v, $\mathrm{KH}_{2} \mathrm{PO}_{4} ; 0.2 \%$, $\mathrm{w} / \mathrm{v}, \mathrm{MgSO}_{4} .7 \mathrm{H}_{2} \mathrm{O} ; 400 \mu \mathrm{g}$ adenine $\mathrm{ml}^{-1}$ ) containing $5 \%$ (w/v) NaCl. S. cerevisiae JBY10 (from Dr Gustin, Rice University, Houston, TX, USA; bog1 1 null mutant, MATa ura3 leu2 his 3 trp1 lys2 ade2 bog1- $1::$ TRP1) and INVSc1 strains (from Invitrogen; MATa bis3-01 leu2 trp1-289 ura352) were cultured aerobically at $30^{\circ} \mathrm{C}$ in YEPD medium $(1 \%$, $\mathrm{w} / \mathrm{v}$, yeast extract; $2 \%, \mathrm{w} / \mathrm{v}$, polypeptone; $2 \%, \mathrm{w} / \mathrm{v}$, glucose). The plate cultures were carried out with YPAD or YEPD plates solidified by $2 \%(\mathrm{w} / \mathrm{v})$ agar and inoculated with $5 \mu \mathrm{l}$ from serial 10 -fold dilutions of stationary-phase cultures. Escherichia coli DH-1 and JM109 strains were cultured aerobically at $37^{\circ} \mathrm{C}$ in LB medium $(1 \%$, w/v, tryptone; $0.5 \%$, $\mathrm{w} / \mathrm{v}$, yeast extract; $1 \%, \mathrm{w} / \mathrm{v}, \mathrm{NaCl} ; \mathrm{pH} 7 \cdot 2)$. The plate cultures were conducted with LB plates solidified by $1.5 \%$ (w/v) agar.

Cloning the $Z$. rouxii gene homologous to the $S$. cerevisiae HOG1 gene. A HOG1-harbouring DNA fragment (approx. 950 bp) was obtained by PCR amplification with oligonucleotides 5' GGAACAAAGGGAAACAGGG $3^{\prime}$ and 5' AGCCGAATAAGGATGAGCCA $3^{\prime}$ as primers and $S$. cerevisiae genomic DNA as the template DNA. After purification by agarose gel electrophoresis, the DNA fragment was labelled with digoxigenin according to the application manual of the Digoxigenin DNA Labelling kit (Boehringer Mannheim) and used as a HOG1 probe.

Z. rouxii genomic DNA was digested with EcoRI, BamHI, HindIII, XbaI, KpnI or SmaI. The digests were electrophoresed on an agarose gel and DNA fragments in the gel were transferred to a nylon filter sheet with a vacuum blotter (Pharmacia Biotech). After fixing the DNA fragment to the nylon filter with a UV-cross-linker, hybridization was performed as described in our previous report (Watanabe et al.,
1995), using a hybridization solution containing the HOG1 probe $\left(30 \mathrm{ng} \mathrm{ml}^{-1}\right)$. The hybridized filter was first washed with $3 \times$ SSC $(1 \times$ SSC contains $150 \mathrm{mM} \mathrm{NaCl}$ and $15 \mathrm{mM}$ sodium citrate), $0 \cdot 2 \%(\mathrm{w} / \mathrm{v})$ SDS, $5 \mathrm{mM}$ EDTA at room temperature and then filter sets were washed under one of the following conditions: (i) $2 \times \mathrm{SSC}, 0 \cdot 2 \%$ (w/v) SDS, $5 \mathrm{mM}$ EDTA, $42{ }^{\circ} \mathrm{C}$; (ii) $0.3 \times \mathrm{SSC}, 0.2 \%$ (w/v) SDS, $5 \mathrm{mM}$ EDTA, $55^{\circ} \mathrm{C}$; (iii) $0.2 \times \mathrm{SSC}, 0.2 \%$ (w/v) SDS, $5 \mathrm{mM}$ EDTA, $55^{\circ} \mathrm{C}$; (iv) $0 \cdot 1 \times$ SSC, $0 \cdot 2 \%(\mathrm{w} / \mathrm{v})$ SDS, $5 \mathrm{mM}$ EDTA, $55^{\circ} \mathrm{C}$. Hybridization of the HOG1 probe was visualized with a Digoxigenin DNA Detection kit (Boehringer Mannheim). Two signals derived from approximately $5 \mathrm{~kb}$ and $6 \mathrm{~kb}$ HindIII DNA fragments were detected under condition (iii).

Z. rouxii genomic DNA was digested with HindIII. DNA fragments sized from 3.5 to $7 \mathrm{~kb}$ were collected and ligated with HindIII-digested charomid 9-36 cosmid DNA. After the in vitro packaging of its ligate, it was transfected to $E$. coli DH-1 cells. The prepared genomic DNA library indicated an efficiency of $2.4 \times 10^{5}$ colonies $\mu \mathrm{g}^{-1}$ for the charomid DNA. Five positive clones were obtained from the library $\left(4 \times 10^{4}\right.$ colonies) by colony hybridization described in a previous report (Watanabe et al., 1995) with the HOG1 probe.

DNA sequencing analysis and transformation of $S$. cerevisiae. Restriction enzyme maps of HindIII DNA fragments were constructed. After subcloning DNA fragments into pUC vectors, nucleotide sequences were determined with a Takara Taq Cycle Sequencing kit (Takara Shuzo) and an autosequencer (DSQ1; Shimadzu). S. cerevisiae cells were transformed according to the application manual of the Alkali Cation Yeast Transformation kit (Bio 101).

Construction of gene-disruption mutants of $Z$. rouxii HOG1 homologous genes (ZrHOG1 and ZrHOG2). Gene disruption was carried out by the one-step gene disruption method (Rothstein, 1983). Briefly, after subcloning the HindIII DNA fragment (ZrHOG1 or ZrHOG2, see Fig. 1) into the pUC19 vector, the recombinant DNA (pUC19-ZrHOG1 or pUC19$\mathrm{ZrHOG2)}$ was digested with EcoRV (two EcoRV sites are present in the HindIII DNA fragment). A LEU2-harbouring DNA fragment, which was isolated by digesting YEp13 with $B g l I I$ and then filled with Klenow fragment, was inserted into EcoRV-digested pUC19-ZrHOG1 or pUC19-ZrHOG2. After digesting pUC19-ZrHOG1 or pUC19-ZrHOG2 with HindIII and removing pUC vector DNA, ZrHOG1- $L L E U 2$ or $Z r H O G 2-\triangle L E U 2$ DNA was used to transform $Z$. rouxii MA11-3 according to the method of Ushio et al. (1988). $\mathrm{Leu}^{+}$ transformants were selected on SC medium without leucine (Rose et al., 1990).

EcoRV-SalI DNA and EcoRV-PstI DNA fragments (see Fig. 1) were isolated from $\mathrm{ZrHOG} 1$ and $\mathrm{ZrHOG} 2$ HindIII DNA fragments, labelled with digoxigenin as described above, and used as ZrHOG1 or ZrHOG2 probes. The gene disruption of ZrHOG1 or $\mathrm{ZrHOG} 2$ was determined by Southern blot analysis with ZrHOG1 or ZrHOG2 probes and genomic DNA prepared from gene-disruption mutants.

Functional expression of ZrHOG1 or ZrHOG2 in S. cerevisiae hog1s null mutants (JBY10 strain). Two HindIII DNA fragments harbouring $\mathrm{ZrHOG} 1$ and $\mathrm{ZrHOG} 2$ were blunted and ligated into NruI-digested YCp50 vector or PvuII-digested YEp13 vector. The recombinant plasmids (YCp50-ZrHOG1, YCp50-ZrHOG2, YEp13-ZrHOG1 and YEp13-ZrHOG2) were used to transform the $S$. cerevisiae JBY10 strain.

Estimation of intracellular glycerol content in various $\boldsymbol{S}$. cerevisiae strains. Various $S$. cerevisiae strains (JBY10, YCp50-ZrHOG1, YCp50-ZrHOG2, YEp13-ZrHOG1, YEp13-ZHO2 and INVSc1 strains) were cultured aerobically 

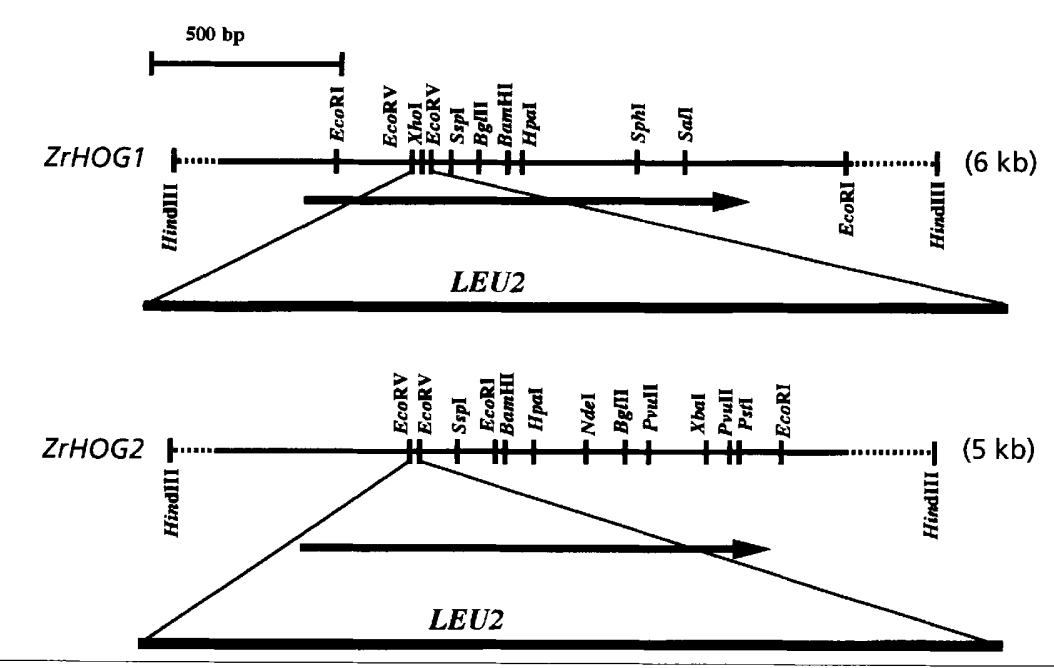

Fig. 1. Restriction map of ZrHOG1 and ZrHOG2 and strategy of gene disruptions. Arrows indicate the loci and orientation of their genes.

\begin{abstract}
Sc HOG1 MTTNEEFIRT QIFGTVFEIT NRYNDLNPVG MGAFGLVCSA TDTLTSOPVA IKRIMKPFST AVLARRTYRE LRLLKBLRHE Ca HOG1 MSADGEFTRT QIFGTVFEIT NRYTELNPVG MGAFGLVCSA VDRLTGQNVA VKKVMRPFST SVLAKRTYRE LRLLRBLKHE $2 x$ HOG1 MATHEEFIRT QVFGTVFEIT KRYTDLNPVG MGAFGLVCSA TDTLAGQPVA IKRIMKPFST AVLAKRTYRE LKLLRHLRHE Zr HOG2 MATHEEFIRT QVFGTVFEIT NRYTDLNPVG MGAFGLVCSA TDTLVGQPVA IRRIMRPFST AVLARRTYRE LRLLRHLRHE

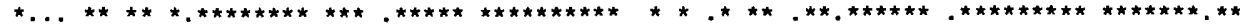

SC-BOG1 NLICLQDIFL SPLEDIYFVT ELQGTDLHRL LQTRPLEKQF VQYFLYQILR GLKYVHSAGV IHRDLRPSNI LINENCDLRI Ca-HOG1 NLITLDDIFI SPLEDIYFVN ELOGTDLHRL LNSRPLEKOF IOYFTYOIMR GLXYIBSAGV IHRDLKPSNI LINENCDLKI Ca-BOG1 NLITLDDIFI SPLEDIYFVN ELQGTDLHRL LNSRPLEKOF IQYFTYQIMR GLKYIHSAGV IHRDLKPSNI LINENCDLKI
Zr-HOG1 NLICLQDIFL SPLEDIYFVT ELQGTDLHRL LQTRPLERQF VQYFLYQILR GLKYVHSAGV IHRDLRPSNI LINENCDLRI $\mathrm{Zr}-$ BOG2 NLICLQDIFL SPLEDIYFVT ELQGTDLHRL LQTRPLEKQF VQYFLYQILR GLKYVHSAGV IHRDLRPSNI LINENCDLRI

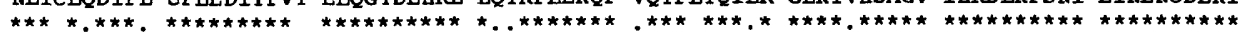

SC-BOG1 CDFGLARIQD POMFGYVSTR YYRAPEIMLT WQKYDVEVDI WSAGCIFAEM IEGKPLFPGK DHVBQFSIIT DLLGSPPKDV Ca-HOG1 CDFGLARLOD PQMTGYVSTR YYRAPEIMLT WQKYDTEVDL WSVGCIIAEM IEGKPLFPGK DEVBQFSIIT ELLGSPPADV Zr-HOG1 CDFGLARIOD POMTGYVSTR YYRAPEIMLT WOKYDVEVDI WSAGCIFSEM IEGKPLFPGK VHVHQFSIIT DLLGSPPRDV Zx-BOG2 CDFGLARIQD PQMTGYVSTR YYRAPEIMET WQRYDVEVDI WSAGCIFSEM IEGKPLFPGK DHVHQFSIIT DLLGSPPRDV

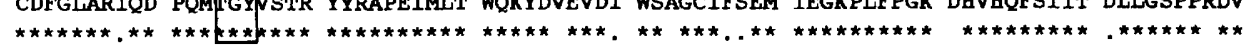

SC-HOG1 INTICSENTL KFVTSLPHRD PIPFSERFRT ---VEPDAVD LLEKMLVFDP KRRITAADAL AHPYSAPYBD PTDEPVADAK Ca-HOG1 IDTICSENTL RFVQSLPERD PIPFSERFAS CTHVEPEAID LIARLLVFDP KKRISAVQGL THPYMEAYHD PTDEPVCESK Zr-HOG1 IITICSEDTL RFVTSLPHRD PVPFQERFRA ---VEPDAVD LLGRMLVFDP KKRITAADAL VHPYLAPYHD PTDEPIAEAO $\mathrm{Zr}$-HOG2 INTICSENTL KFVTSLPHRD PVPFOERFKT ---VEPDAVD LLRRMLVFVP KKRITAADAL VHPYLAPYHD PTDEPTAEAQ

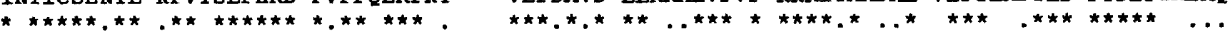

SC-BOG1 FDWBFNDADL PVDTWRVMMY SEILDFHKIG $(+69)$

Ca-HOG1 FDWSFNDADL PVDTWRVMMY SEILDFBQTV $(+27)$

ZI-HOG1 FDWDFNDADL PVDTWRVMMY SEILDFHKIG $(+60)$

ZI-HOG2 FDWDFNDADL PVDTWRVMMY SEILDFEKIG $(+73)$

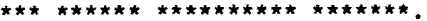

Fig. 2. Homology alignment of amino acid sequences of Hog1ps from S. cerevisiae (Sc-HOG1), C. albicans (Ca-HOG1) and $Z$. rouxii ( $\mathrm{Zr}$-HOG1 and $\mathrm{Zr}$-HOG2). Identical residues and conservative substitutions are shown by asterisks and dots, respectively. The TGY motif is boxed.

at $30^{\circ} \mathrm{C}$ in the absence of $\mathrm{NaCl}$. Cells grown to the early exponential phase $\left(\mathrm{OD}_{600} 0 \cdot 5\right)$ were collected and subjected to a new medium containing $0.5 \mathrm{M} \mathrm{NaCl}$. After incubation for an appropriate period, cells were harvested and washed twice with $0.5 \mathrm{M} \mathrm{NaCl}$. The cell pellet was then suspended in $5 \mathrm{ml}$ distilled water and disrupted by vortexing for $10 \mathrm{~min}$ with $5 \mathrm{~g}$ glass beads at $4{ }^{\circ} \mathrm{C}$. The homogenate was centrifuged for $20 \mathrm{~min}$ at $10000 \mathrm{~g}$ at $4{ }^{\circ} \mathrm{C}$. The supernatant was immediately heated for $10 \mathrm{~min}$ in boiling water to inactivate enzymes connected to the metabolism of glycerol. After centrifugation for $20 \mathrm{~min}$ at $10000 \mathrm{~g}$ to remove denatured protein, the supernatant was used to measure the intracellular glycerol concentration. The glycerol content was determined according to the application manual of the Glycerol-F kit (Bochringer Mannheim).

Northern blot analysis of GPD1 mRNA in $S$. cerevisiae ZrHOG1- or ZrHOG2-harbouring strains. Total RNA was prepared from YCp50-ZrHOG1 and YCp50-ZrHOG2 strains according to a method described previously (Watanabe $e t$ al., 1995). The DNA fragment that encodes the GPD1 of $S$. cerevisiae was prepared by PCR amplification with oligonucleotides 5' ATGTCTGCTGCTGCTGATAG 3' and 5' AGAGCCTCTGAAAAAAGTGGG $3^{\prime}$ as primers and genomic DNA as a template. The DNA fragment was labelled with digoxigenin as described above and used as a GPD1 probe. Northern blot analysis was carried out as described previously (Watanabe et al., 1993).

\section{RESULTS}

\section{Cloning of HOG1 homologues from Z. rouxii}

A Z. rouxii genomic DNA library was constructed as described in Methods. By screening 40000 independent clones with a HOG1 probe, five positive clones were 

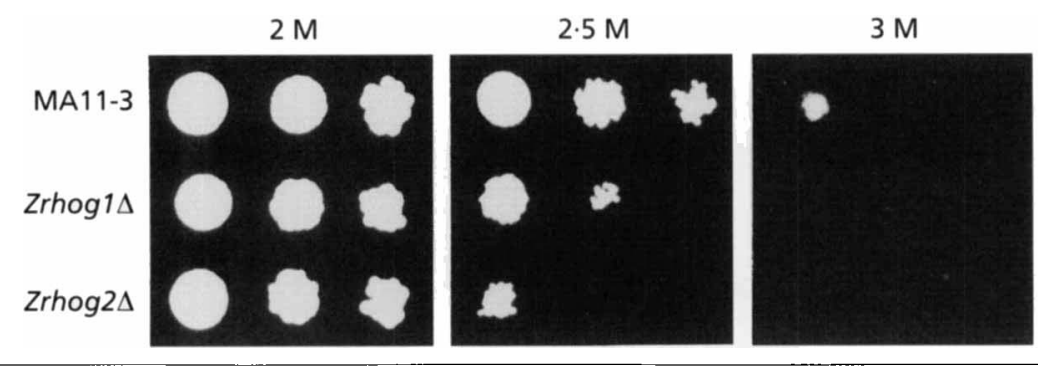

Fig. 3. Growth of $Z$. rouxii wild-type (MA113) and $Z r h o g 1 \Delta$ and $Z r h o g 2 \Delta$ null mutant strains. Serial 10 -fold dilutions of saturated cultures were spotted onto YPAD plates supplemented with $\mathrm{NaCl}$ as indicated.
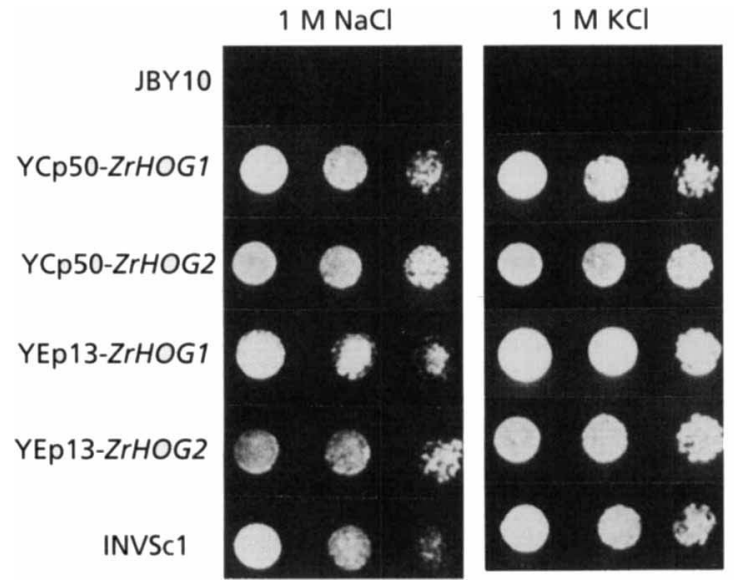

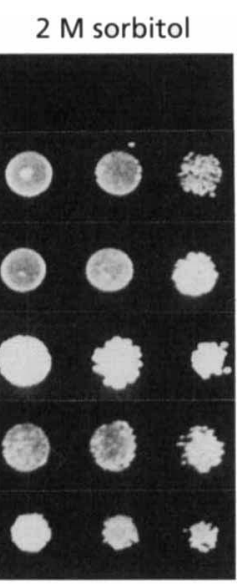

Fig. 4. Growth of S. cerevisiae hog1s (JBY10), wild-type (INVSC1) and ZrHOG1- or ZrHOG2-harbouring (YCp50-ZrHOG1, YCp50ZrHOG2, YEp13-ZrHOG1 or YEp13-ZrHOG2) strains in the presence of $\mathrm{NaCl}_{x} \mathrm{KCl}$ or sorbitol. Serial 10 -fold dilutions of saturated cultures were spotted onto YEPD plates supplemented with $\mathrm{NaCl}, \mathrm{KCl}$ or sorbitol as indicated. obtained. Restriction enzyme maps of HindIII DNA fragments present in cosmid DNAs recovered from the clones were constructed. By comparing their maps, the five clones were divided into two groups based on the size of their HindIII DNA fragments (Fig. 1). One group (designated ZrHOG1) had $6 \mathrm{~kb}$ HindIII DNA fragments and the other group ( $\mathrm{ZrHOG}$ ) had $5 \mathrm{~kb}$ fragments.

The nucleotide sequence of the region hybridized with the HOG1 probe was determined for the two groups. ORFs that lacked introns were found in both groups. The nucleotide sequences of $\mathrm{ZrHOG1}$ and $\mathrm{ZrHOG} 2$ indicated approximately 70 and $80 \%$ homology to that of S. cerevisiae HOG1 (Brewster et al., 1993). The deduced amino acid sequences of $\mathrm{ZrHog} 1 \mathrm{p}$ and ZrHog2p were approximately 93 and $89 \%$ homologous to that of Hog1p (Brewster et al., 1993; Fig. 2). These results indicate that genes homologous to $S$. cerevisiae HOG1 are present in $Z$. rouxii cells and the copy number of HOG1 homologue in $Z$. rouxii is different from that in $S$. cerevisiae. The TGY motif (Brewster et al., 1993) was found in the $\mathrm{ZrHog} 1 \mathrm{p}$ and $\mathrm{ZrHog} 2 \mathrm{p}$ sequences.

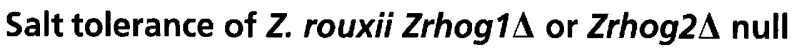 mutants}

The disruption of $\mathrm{ZrHOG} 1$ or $\mathrm{ZrHOG} 2$ in $Z$. rouxii cells was carried out as described in Methods. Both disruptions were verified by Southern blot analysis of genomic DNA prepared from $Z$. rouxii Zrhog1s and

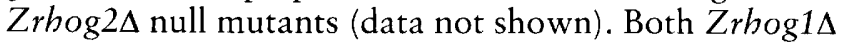
and $Z$ rhog $2 \Delta$ null mutants could grow in the presence of $\mathrm{NaCl}$ below $2.5 \mathrm{M}$ (Fig. 3). In contrast, the parental

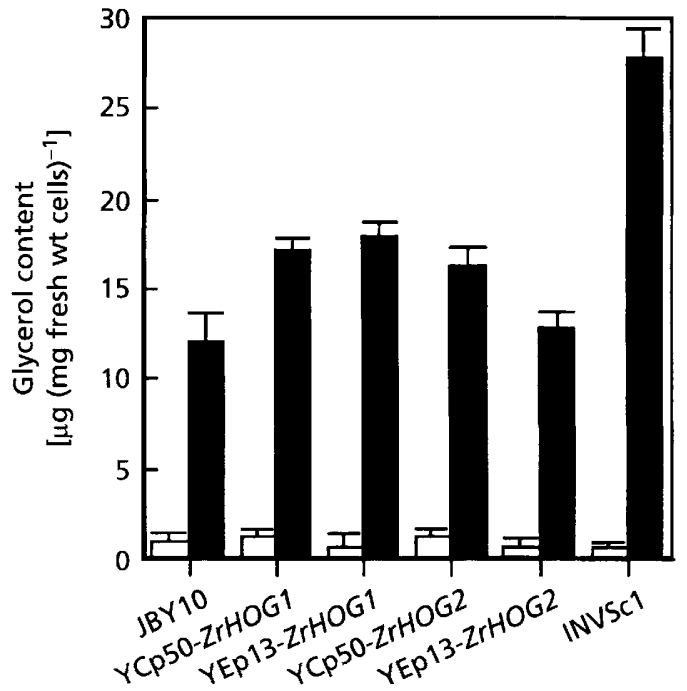

Fig. 5. Changes in intracellular content of glycerol in $S$. cerevisiae hog1D (JBY10), wild-type (INVSC1) and ZrHOG1- or ZrHOG2-harbouring (YCp50-ZrHOG1, YCp50-ZrHOG2, YEp13ZrHOG1 or YEp13-ZrHOG2) strains. Cells were grown in the absence of $\mathrm{NaCl}$ and harvested at $\mathrm{OD}_{600} 0.5$. The cells were resuspended in fresh medium containing $0.5 \mathrm{M} \mathrm{NaCl}$ at the same cell concentration and incubated at $30^{\circ} \mathrm{C}$ for $1 \mathrm{~h}$, and then glycerol content was determined. Results are the means of at least three independent experiments. The standard errors of means are indicated. $\square, \mathrm{NaCl}$-free; $\square, 0.5 \mathrm{M} \mathrm{NaCl}$.

MA11-3 strain was able to grow in the presence of $3 \mathrm{M}$ $\mathrm{NaCl}$ (Fig. 3). This indicates that the salt tolerance of $Z$. rouxii cells decreases when $\mathrm{ZrHOG1}$ or $\mathrm{ZrHOG} 2$ was 

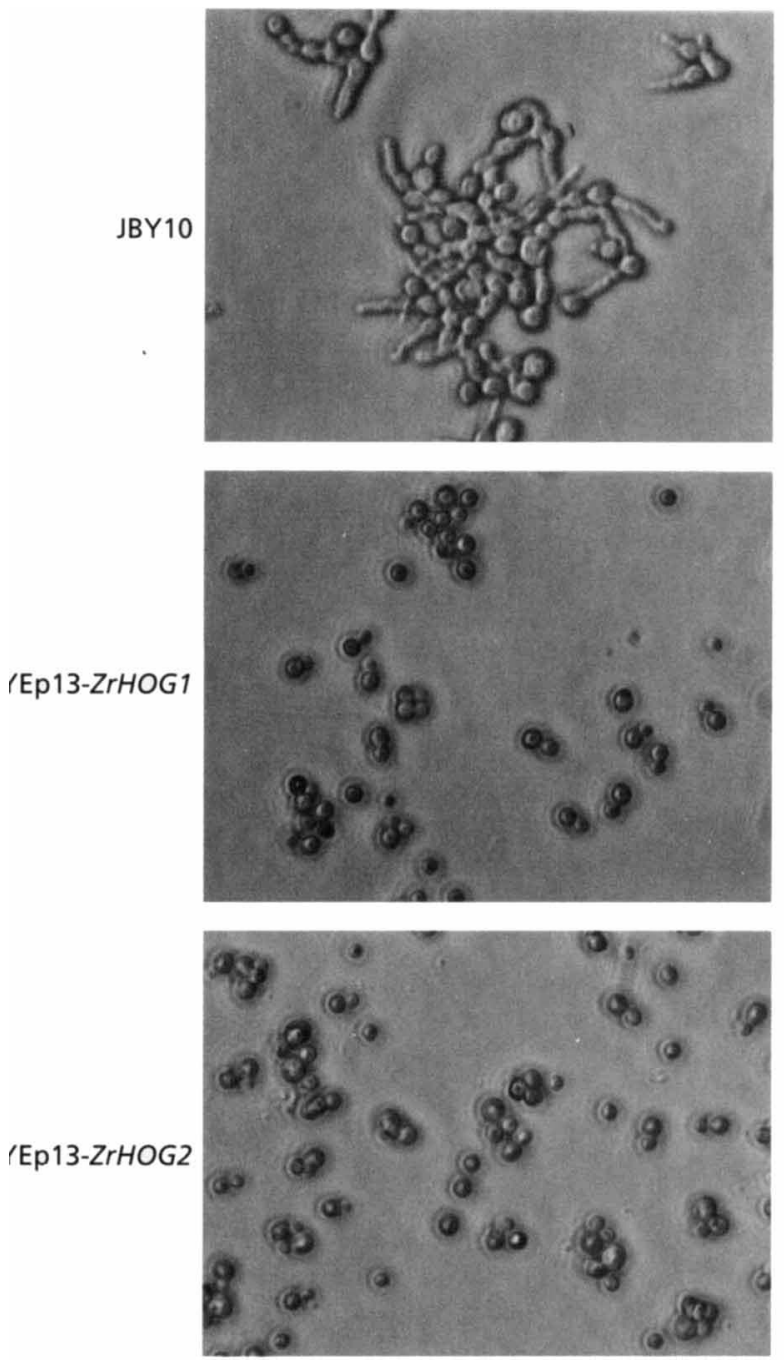

YCp50-ZrHOG1
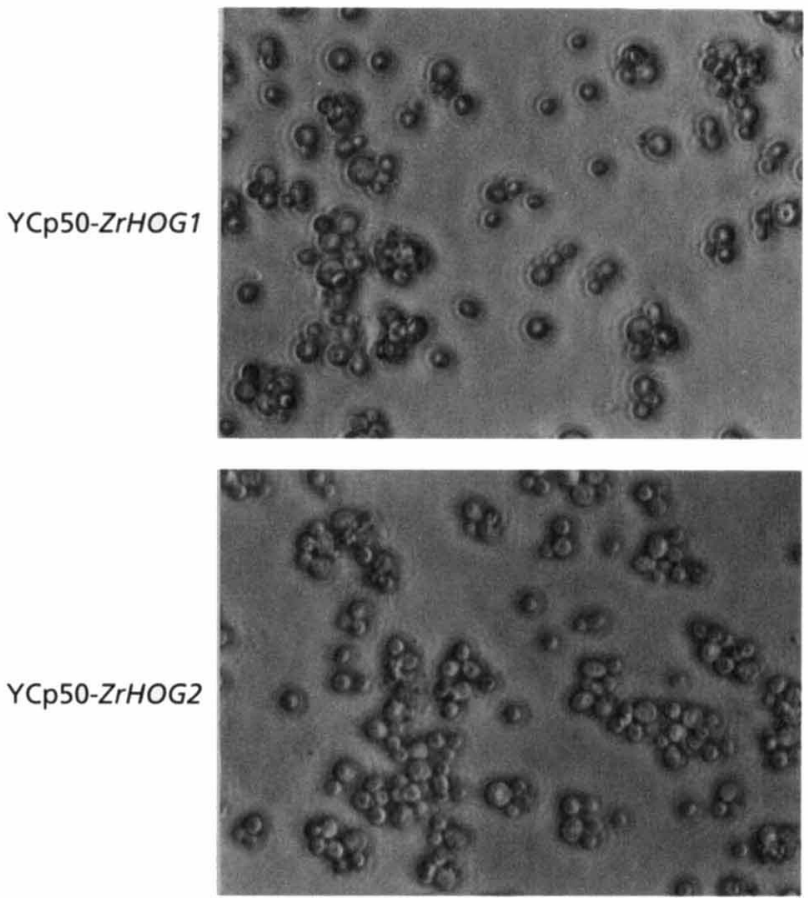

Fig. 6. Morphology of S. cerevisiae hog1A (JBY10) and ZrHOG1- and ZrHOG2-harbouring (YCp50-ZrHOG1, YCp50ZrHOG2, YEp13-ZrHOG1 or YEp13-ZrHOG2) strains. Cells were grown in the presence of $0.5 \mathrm{M} \mathrm{NaCl}$.

disrupted, and both $\mathrm{ZrHog} 1 \mathrm{p}$ and $\mathrm{ZrHog} 2 \mathrm{p}$ are presumed to participate functionally in the transmission pathway of the osmotic signal in $Z$. rouxii cells.

\section{Complementation of an S. cerevisiae hog1 $1 \Delta$ null mutant by ZrHOG1 and ZrHOG2}

To elucidate the functions of $\mathrm{ZrHOG1}$ and $\mathrm{ZrHOG}$, complementation of an $S$. cerevisiae hog $1 \Delta$ null mutant (JBY10 strain) was tested with ZrHOG1 and ZrHOG2. The salt tolerance of the JBY10 strain is lower than that of the wild-type strain, because the enhancement of GPDH activity in response to osmotic stress is low when there is no MAP kinase gene (HOG1). If $\mathrm{ZrHOG1}$ or ZrHOG2 complemented the deletion of HOG1 in the $S$. cerevisiae JBY10 strain, the osmotic tolerance of the recombinant strain should increase when compared to the JBY10 strain. This transcomplementation has been used to analyse the function of MAP kinase genes cloned from mammalian and plant cells (Galcheva-Gargova et al., 1994; Han et al., 1994; Kumer et al., 1995; Popping et al., 1996). DNA fragments (HindIII fragments, see Fig. 1) which harbour the promoters, ORFs and terminators of $\mathrm{ZrHOG} 1$ and $\mathrm{ZrHOG} 2$ genes were inserted into the yeast centromeric vector YCp50 and $2 \mu$-circle-based vector YEp13, and the recombinant plasmid DNAs were transformed into the JBY10 strain. The transformed yeast strains (YCp50-ZrHOG1, YCp50-ZrHOG2, YEp13-ZrHOG1 and YEp13$\mathrm{ZrHOG} 2$ strains) were plated on plates containing $1 \mathrm{M}$ $\mathrm{NaCl}, 1 \mathrm{M} \mathrm{KCl}$ or $2 \mathrm{M}$ sorbitol (Fig. 4). The hog1s null mutant (JBY10 strain) could not grow on these plates, but YCp50-ZrHOG1, YCp50-ZrHOG2, YEp13ZrHOG1 and YEp13-ZrHOG2 strains indicated growth that was comparable to the wild-type strain (INVSc1) under all conditions. These results indicate that both $Z r H O G 1$ and $Z r H O G 2$ have functions similar to the $S$. cerevisiae MAP kinase gene (HOG1) and that at least 


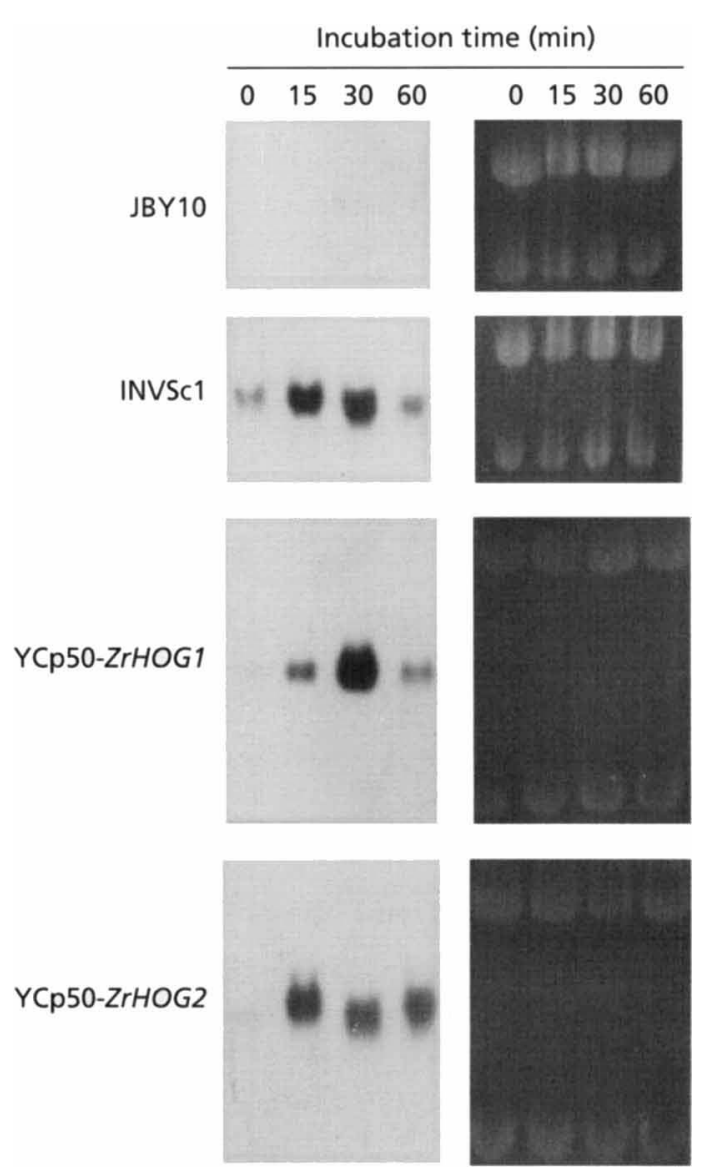

Fig. 7. Northern blot analysis of the GPD1 transcript in S. cerevisiae hog1 $\triangle$ (JBY10), wild-type (INVSC1) and ZrHOG1- and ZrHOG2-harbouring (YCp50-ZrHOG1 and YCp50-ZrHOG2) strains. Cells subjected to the $\mathrm{NaCl}$ treatment indicated in Fig. 5 were cultivated at $30^{\circ} \mathrm{C}$. Samples of cells were withdrawn from cultures at the intervals indicated. Total RNA (approx. $8 \mu \mathrm{g}$ ) was loaded in each lane. An ethidium bromide gel revealing the $26 \mathrm{~S}$ and 185 rRNA bands is shown to validate equal loading of the lanes and the integrity of the RNAs.

the promoters and terminators of $Z r H O G 1$ and $\mathrm{ZrHOG} 2$ are functional in S. cerevisiae cells.

In S. cerevisiae hog1s null mutants (JBY10 strain), the enhancement in intracellular glycerol content during osmotic stress was much lower (Fig. 5) than in the wildtype strain (INVSc1). When the JBY10 strain, YCp50ZrHOG1 and YCp50-ZrHOG2 strains and YEp13$\mathrm{ZrHOG} 1$ and YEp13-ZrHOG2 strains were cultured in the presence of $0.5 \mathrm{M} \mathrm{NaCl}, Y C p 50-Z r H O G 1$ and YCp50-ZrHOG2 strains and the YEp13-ZrHOG1 strain showed a significant increase in glycerol content compared with the JBY10 strain (Fig. 5). The YEp13ZrHOG2 strain showed only a slight increase in glycerol content (Fig. 5). However, these increased levels of glycerol were lower than that observed in the INVSc1 strain. This difference may result from the lower promoter activity of $\mathrm{ZrHOG1}$ or $\mathrm{ZrHOG} 2$ in $S$. cerevisiae cells. Also, no effect of increased ZrHOG1 copy number on glycerol content was observed, and increased $\mathrm{ZrHOG} 2$ copy number negatively affected the production of glycerol. The cause of the negative effect of increases in $\mathrm{ZrHOG} 2$ copy number is unclear at the present.

It has been previously reported that $S$. cerevisiae hog1 $1 \Delta$ null mutants have an aberrant cell morphology under osmotic stress, being large, multinucleated cells with multiple elongated buds (Brewster et al., 1993). The $S$. cerevisiae hog1 $1 \Delta$ null mutant JBY10 was similar to this, but a hog1s mutant harbouring YEp13-ZrHOG1, YEp13-ZrHOG2, YCp50-ZrHOG1 or YCp50ZrHOG2 showed normal cell shape (Fig. 6). This indicates that the abnormal cell shape of hog1 $1 \Delta$ null mutants can be rescued by the expression of $\mathrm{ZrHOG1}$ or ZrHOG2.

\section{Transcription of GPD1 in S. cerevisiae cells by expressing ZrHOG1 or ZrHOG2}

As shown above, the expression of $\mathrm{ZrHOG1}$ or $\mathrm{ZrHOG}$ enhanced the intracellular glycerol accumulation in $S$. cerevisiae hog $1 \Delta$ null mutants. To clarify whether $\mathrm{ZrHog} 1 \mathrm{p}$ and $\mathrm{ZrHog} 2 \mathrm{p}$ are functionally equivalent to Hog $1 p$ derived from S. cerevisiae (Albertyn et al., 1994) in regulating GPD1 transcription in $S$. cerevisiae, Northern blot analysis on GPD1 was carried out in S. cerevisiae YCp50-ZrHOG1 and YCp50$\mathrm{ZrHOG} 2$ strains. After $\mathrm{NaCl}$ shock the transcription of GPD1 in ZrHOG1- and ZrHOG2-harbouring strains was induced markedly, to the same level as the $S$. cerevisiae wild-type INVSc1 strain, but the $S$. cerevisiae hog1s null mutant (JBY10 strain) failed to enhance the transcription of GPD1 under the same conditions (Fig. 7). This indicates that $\mathrm{ZrHog} 1 \mathrm{p}$ and $\mathrm{ZrHog} 2 \mathrm{p}$ have the same function as Hog $1 \mathrm{p}$ and can regulate the transcription of GPD1 in $S$. cerevisiae. Only a slight difference between YCp50-ZrHOG1 and YCp50$\mathrm{ZrHOG} 2$ strains was observed. This was that the induction of GPD1 transcription in the YCp50-ZrHOG1 strain was maximal at 30 min after $\mathrm{NaCl}$ shock and then diminished by $60 \mathrm{~min}$, similar to that observed in the wild-type INVSc1 strain (Fig. 7); in contrast, the transcription of GPD1 in the YCp50-ZrHOG2 strain remained at the maximum level for $60 \mathrm{~min}$ (Fig. 7). This may indicate that the regulation by $\mathrm{ZrHog} 1 \mathrm{p}$ or $\mathrm{ZrHog} 2 \mathrm{p}$ is different during the induction of transcription of GPD1 in $S$. cerevisiae cells.

\section{DISCUSSION}

\section{Cloning of putative MAP kinase genes (ZrHOG1 and ZrHOG2) from $Z$. rouxii}

When S. cerevisiae cells are exposed to a high level of osmotic shock, a signal transmission pathway of osmotic stress, called the MAP kinase cascade (HOG pathway), is activated. This leads to elevated production of glycerol by GPDH and intracellular glycerol accumulated. The HOG1 gene product of $S$. cerevisiae is the key element in 
the HOG pathway, and activated Hog1p has been reported to phosphorylate the transcriptional regulator for the GPDH gene (GPD1; Albertyn et al., 1994). Genes homologous to HOG1 have been identified in the yeasts Candida albicans (San Jose et al., 1996) and Schizosaccharomyces pombe (Degols et al., 1996; Kato et al., 1996). In the salt-tolerant yeast $Z$. rouxii, also, glycerol is the main compatible osmolyte used to adjust osmotic imbalance (Reed et al., 1987). It has been assumed that a pathway similar to the HOG pathway might be present in $Z$. rouxii cells. Thus, in the present study, we cloned genes homologous to $S$. cerevisiae HOG1 to help characterize the signal transmission pathway of osmotic stress in $Z$. rouxii.

Two genes ( $\mathrm{ZrHOG1}$ and $\mathrm{ZrHOG}$ ) homologous to HOG1 were identified in $Z$. rouxii. The deduced amino acid sequences encoded by $\mathrm{ZrHOG} 1$ and $\mathrm{ZrHOG} 2$ were highly homologous to that encoded by HOG1 (Fig. 2). Moreover, the TGY motif was found in the sequences of $\mathrm{ZrHog} 1 \mathrm{p}$ and $\mathrm{ZrHog} 2 \mathrm{p}$ (Fig. 2). This motif was indicated to be phosphorylated by MAP kinase kinase (Pbs2p; Boguslawski, 1992) in S. cerevisiae (Brewster et al., 1993). If a kinase similar to Pbs2p is present in $Z$. rouxii cells, both $\mathrm{ZrHog} 1 \mathrm{p}$ and $\mathrm{ZrHog} 2 \mathrm{p}$ should be phosphorylated by the kinase.

\section{Functional expression of ZrHOG1 and ZrHOG2 in S. cerevisiae}

When ZrHOG1 and $\mathrm{ZrHOG} 2$ were introduced with a centromeric vector, YCp50, and $2 \mu$-circle-based vector, YEp13, into an $S$. cerevisiae hog1s null mutant, $\mathrm{ZrHOG1}$ and $\mathrm{ZrHOG} 2$ were found to complement the phenotypes (salt tolerance, osmotic tolerance, glycerol content and abnormal cell shape) of the mutant (Figs 4, 5 and 6), indicating that the products encoded by $\mathrm{ZrHOG} 1$ and $\mathrm{ZrHOG} 2$ are functional as MAP kinase in $S$. cerevisiae cells. This strongly suggests that there is an equivalent $\mathrm{HOG}$ pathway in $Z$. rouxii. The defect in $\mathrm{NaCl}$-induced GPD1 transcription in an $S$. cerevisiae hog1 $1 \Delta$ null mutant was corrected by $Z r H O G 1$ and ZrHOG2. The transcription of GPD1 in the ZrHOG1harbouring mutant was transient, as it was in wild-type strains of $S$. cerevisiae, but the $\mathrm{ZrHOG2-harbouring}$ mutant showed a prolonged transcription of GPD1 (Fig. 7). This prolonged transcription of GPD1 may be significant in relation to glycerol production in $Z$. rouxii cells. However, since GPDH of $Z$. rouxii was not studied here, the regulation by $\mathrm{ZrHog} 1 \mathrm{p}$ or $\mathrm{ZrHog} 2 \mathrm{p}$ of the GPDH gene transcription in $Z$. rouxii subjected to osmotic shock remains to be elucidated. The observed differences in GPD1 transcription in S. cerevisiae could be explained by a difference in the actions of $\mathrm{ZrHog} 1 \mathrm{p}$ and $\mathrm{ZrHog} 2 \mathrm{p}$ on the transcriptional regulators (e.g. Msn2p or Msn4p; Inoue et al., 1998) of GPD1. One possibility is that $\mathrm{ZrHog} 1 \mathrm{p}$ and $\mathrm{ZrHog} 2 \mathrm{p}$ regulate different regulators. Since a negative effect of increases in copy number on glycerol production was observed only in the $\mathrm{ZrHOG2}$-harbouring strain (Fig. 5), this seems likely.

\section{Decreased salt tolerance of $Z$. rouxii Zrhog1 $\Delta$ and Zrhog2 $\Delta$ null mutants}

The possibility that the functions of both the products encoded by $Z r H O G 1$ and $Z r H O G 2$ are related to the salt tolerance of $Z$. rouxii cells was indicated by the analysis of phenotypes of $Z$. rouxii $Z$ rhog1s and Zrhog $2 \Delta$ null mutants (Fig. 3). Because the GPDH gene of $Z$. rouxii has not been cloned, we could not research the functions of $Z r H O G 1 / 2$ in detail in $Z$. rouxii. Their functions will be reported elsewhere, including the construction of ZrHOG1 and $\mathrm{ZrHOG} 2$ double disruption mutants and the determination of glycerol content in $Z$. rouxii Zrhog1s or Zrhog $2 \Delta$ null mutants. In $S$. cerevisiae, activated $H \operatorname{Hog} 1 \mathrm{p}$ has been reported to regulate the transcription of genes encoding stressinduced proteins other than GPDH. For example, the expression of ENA1, which encodes $\mathrm{Na}^{+}$-ATPase and is related mainly to the efflux of $\mathrm{Na}^{+}$in $S$. cerevisiae, is regulated by $\mathrm{Hog} 1 \mathrm{p}$ at low $\mathrm{NaCl}$ concentrations $(0.3 \mathrm{M})$ and by calcineurin/calmodulin at higher concentrations (0.8 M; Marquez \& Serrano, 1996). Also, the transcription of GLO1 encoding glyoxalase $\mathrm{I}$ is regulated by Hog1p in S. cerevisiae (Inoue et al., 1998). Therefore, it is likely that $\mathrm{ZrHog} 1 \mathrm{p}$ and $\mathrm{ZrHog} 2 \mathrm{p}$ could regulate the expression of genes related to ion homeostasis and other functions, if there is a relationship to the salt tolerance of $Z$. rouxii cells.

The present study revealed that at least two functional putative MAP kinase genes ( $\mathrm{ZrHOG1}$ and $\mathrm{ZrHOG}$ ) are present and both of their products are functional in Z. rouxii. There is only one HOG1 MAP kinase gene in other yeasts (Brewster et al., 1993; San Jose et al., 1996; Degols et al., 1996; Kato et al., 1996), thus the two genes seem specific to $Z$. rouxii. This may suggest that two HOG1 homologues play crucial roles in the salt tolerance (osmotic tolerance) of $Z$. rouxii cells.

\section{ACKNOWLEDGEMENTS}

We wish to express thanks to M. C. Gustin for his generous gift of the S. cerevisiae JBY10 strain and to K. Ushio for his generous gift of the $Z$. rouxii MA11-3 strain.

\section{REFERENCES}

Akhtar, N., Blomberg, A. \& Adler, L. (1997). Osmoregulation and protein expression in a pbs $2 \Delta$ mutant of Saccharomyces cerevisiae during adaptation to hypersaline stress. FEBS Lett 403, 173-180.

Albertyn, J., Homann, A., Thevelein, J. M. \& Prior, B. A. (1994). GPD1, which encodes glycerol-3-phosphate dehydrogenase, is essential for growth under osmotic stress in Saccharomyces cerevisiae, and its expression is regulated by the high-osmolarity glycerol response pathway. Mol Cell Biol 14, 4135-4144.

Boguslawski, G. (1992). PBS2, a yeast gene encoding a putative protein kinase, interacts with the RAS2 pathway and affects osmotic sensitivity of Saccharomyces cerevisiae. J Gen Microbiol 138, 2425-2432.

Brewster, J. L., de Valoir, T., Dwyer, N. D., Winter, E. \& Gustin, M. C. (1993). An osmosensing signal transduction pathway in yeast. Science 259, 1760-1763. 
Degols, G., Shiozaki, K. \& Russell, P. (1996). Activation and regulation of the Spc1 stress-activated protein kinase in Schizosaccharomyces pombe. Mol Cell Biol 16, 2870-2877.

Galcheva-Gargova, Z., Derijard, B., Wu, L.-H. \& Davis, R. J. (1994). An osmosensing signal transduction pathway in mammalian cells. Science 265, 806-808.

Han, J., Lee, J.-D., Bibbs, L. \& Ulevitch, R. J. (1994). A MAP kinase targeted by endotoxin and hyperosmolarity in mammalian cells. Science 265, 808-811.

Inoue, Y., Tsujimoto, Y. \& Kimura, A. (1998). Expression of the glyoxalase I gene of Saccharomyces cerevisiae is regulated by high osmolarity glycerol mitogen-activated protein kinase pathway in osmotic stress response. J Biol Chem 273, 2977-2983.

Kato, T., Jr, Okazaki, K., Murakami, H., Stettler, S., Fonyes, P. A. \& Okayama, H. (1996). Stress signal, mediated by a Hog1-like MAP kinase, controls sexual development in fission yeast. FEBS Lett 378, 207-212.

Kumer, S., McLaughlin, M. M., McDonnell, P. C., Lee, J. C., Livi, G. P. \& Young, P. R. (1995). Human mitogen-activated protein kinase CSBP1, but not CSBP2, complements a hog1 deletion in yeast. J Biol Chem 270, 29043-29046.

Maeda, T., Wurgler-Murphy, S. M. \& Saito, H. (1994). A twocomponent system that regulates an osmosensing MAP kinase cascade in yeast. Nature 369, 242-245.

Maeda, T., Takekawa, M. \& Saito, H. (1995). Activation of yeast PBS2 MAPKK by MAPKKKs or by binding of an SH3-containing osmosensor. Science 269, 554-558.

Marquez, J. A. \& Serrano, R. (1996). Multiple transduction pathways regulate the sodium-extrusion gene PMR2/ENA1 during salt stress in yeast. FEBS Lett 382, 89-92.

Norbeck, J. \& Blomberg, A. (1997). Metabolic and regulatory changes associated with growth of Saccharomyces cerevisiae in $1.4 \mathrm{M} \mathrm{NaCl}$. J Biol Chem 272, 5544-5554.

Ota, I. M. \& Varshavsky, A. (1993). A yeast protein similar to bacterial two-component regulators. Science 262, 566-569.

Popping, B., Gibbons, T. \& Watson, M. D. (1996). The Pisum sativum MAP kinase homologue (PsMAPK) rescues the Saccharo- myces cerevisiae hog1 deletion mutant under conditions of high osmotic stress. Plant Mol Biol 31, 355-363.

Posas, F. \& Saito, H. (1997). Osmotic activation of the HOG MAPK pathway via Ste11p MAPKKK: scaffold role of Pbs2p MAPKK. Science 276, 1702-1705.

Posas, F., Wurgler-Murphy, S. M., Maeda, T., Witten, E. A., Thai, T. C. \& Saito, H. (1996). Yeast HOG1 MAP kinase cascade is regulated by a multistep phosphorelay mechanism in the SLN1YPD1-SSK1 two-component osmosensor. Cell 86, 865-875.

Reed, R. H., Chudek, J. A., Foster, R. \& Gadd, G. M. (1987). Osmotic significance of glycerol accumulation in exponentially growing yeasts. Appl Environ Microbiol 53, 2119-2123.

Rose, M. D., Winston, F. \& Hieter, P. (1990). Methods in Yeast Genetics: a Laboratory Course Manual. Cold Spring Harbor, NY: Cold Spring Harbor Laboratory.

Rothstein, R. J. (1983). One-step gene disruption in yeast. Methods Enzymol 101, 202-211.

San Jose, C., Alonso Monge, R., Perez-Diaz, R., Pla, J. \& Nombela, C. (1996). The mitogen-activated protein kinase homolog HOG1 gene controls glycerol accumulation in the pathogenic fungus Candida albicans. J Bacteriol 17, 5850-5852.

Schuller, C., Brewster, J. L., Alexander, M. R., Gustin, M. C. \& Ruis, H. (1994). The HOG pathway controls osmotic regulation of transcription via the stress response element (STRE) of the Saccharomyces cerevisiae CTT1 gene. EMBO J 13, 4382-4389.

Ushio, K., Tatsumi, H., Araki, H., Toh-e, A. \& Oshima, Y. (1988). Construction of a host-vector system in the osmophilic haploid yeast Zygosaccharomyces rouxii. J Ferment Technol 66, 481-488.

Watanabe, Y., Sanemitsu, Y. \& Tamai, Y. (1993). Expression of plasma membrane proton-ATPase gene in salt-tolerant yeast Zygosaccharomyces rouxii is induced by sodium chloride. FEMS Microbiol Lett 114, 105-108.

Watanabe, Y., Miwa, S. \& Tamai, Y. (1995). Characterization of $\mathrm{Na}^{+} / \mathrm{H}^{+}$-antiporter gene closely related to the salt-tolerance of yeast Zygosaccharomyces rouxii. Yeast 11, 829-838.

Received 27 May 1998; revised 11 August 1998; accepted 17 September 1998. 